\title{
Proximal humerus fractures (PHFs): comparison of functional outcome 1 year after minimally invasive plate osteosynthesis (MIPO) versus open reduction internal fixation (ORIF)
}

\author{
Laura Buchmann ${ }^{1,2} \cdot$ Esther M. M. van Lieshout ${ }^{3} \oplus \cdot$ Miliaan Zeelenberg $^{3} \cdot$ Dennis den Hartog $^{3}(1) \cdot$

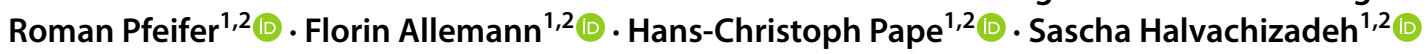

Received: 15 March 2021 / Accepted: 11 June 2021 / Published online: 3 July 2021

(C) The Author(s) 2021

\begin{abstract}
Purpose Osteosynthetic treatment strategies of PHFs include MIPO or ORIF techniques. The aim of this study was to compare the 1 year outcome following either technique in type B PHFs.

Methods This study was designed as a retrospective cohort study of patients treated at one academic Level 1 trauma center. Patients from 2009 to 2019 who required surgical treatment of a type B PHF were eligible to be included in this study. Patients with A- or C-type fractures or patients requiring arthroplasty were excluded. All patients were treated with Proximal Humerus Interlocking System (PHILOS) and stratified according the approach into Group MIPO or Group ORIF. Outcome measures include local complications that occurred during hospitalization, nonunion after 12 months, and range of motion after 1 year follow-up.

Results This study included 149 (75.3\%) patients in Group ORIF, and 49 (24.7\%) in Group MIPO. The fracture morphology and concomitant injuries were comparable amongst these groups. When compared with Group MIPO, Group ORIF had a 2.6 (95\% CI 0.6-11.7) higher risk of suffering from local complications. The rate of postoperative nerve lesions was comparable (OR 0.9, 95\% CI 0.1-9.7) as was the rate of soft tissue complications (OR 2.0, 95\% CI 0.2-17.2). The risk for nonunion was 4.5 times higher $(95 \%$ 1.1-19.5) in Group ORIF when compared with Group MIPO. Group MIPO had a higher chance of flexion above $90^{\circ}$ (OR 8.2, 95\% CI 2.5-27.7).

Conclusion This study provides indications that patients following surgical treatment of PHFs in MIPO technique might have favourable outcome. Large-scale and high-quality studies are warranted to confirm these results.
\end{abstract}

Keywords MIPO $\cdot$ ORIF $\cdot$ PHILOS $\cdot$ Proximal humerus fracture $\cdot$ Soft tissue $\cdot$ Osteosynthesis

\section{Purpose}

PHFs account for approximately $5 \%$ of all fractures in adult patients and are in the top three causes of osteoporotic fractures [1]. The increasing incidence of PHFs might be

Sascha Halvachizadeh

Sascha.Halvachizadeh@usz.ch

1 Department of Trauma, University Hospital Zurich, Raemistrasse 100, 8091 Zurich, Switzerland

2 Faculty of Medicine, University of Zurich, Raemistrasse 75, 8006 Zurich, Switzerland

3 Trauma Research Unit, Department of Surgery, Erasmus MC, University Medical Center Rotterdam, Rotterdam, Netherlands explained by an increased life expectancy and more active lifestyle $[2,3]$. The treatment strategy depends on the fracture morphology, activity level of the patients, and the soft tissue condition [4]. Non- or minimally displaced fractures qualify for potential non-operative treatment strategies [5]. Patients suffering from displaced fractures might, however, benefit from a surgical treatment strategy to improve shoulder function [6]. In general, the major surgical treatment strategies for PHFs include osteosynthesis and arthroplasty [7]. The proximal humeral interlocking system (PHILOS) has been reported among the preferred surgical modality in fractures that might benefit from osteosynthesis [8, 9]. However, to improve functional outcome, complications based on unthoughtful management of the soft tissue status, including infection, delayed union, or avascular head necrosis should be reduced [10]. Among others, the MIPO technique has 
gained popularity as a potential surgical treatment strategy for PHFs [11]. Several studies reported beneficial outcomes following MIPO, including the lower rate of vascular damage, shorter duration of surgery and reduced postoperative pain [12-14].

There still is ongoing discussion on the benefits of MIPO when compared with ORIF in type B fractures. Therefore, the aim of this study was to test the following hypothesis: MIPO results in lower complications rates and improved functional outcome 1 year after surgical treatment of type B PHFs.

\section{Methods}

This study was designed as a retrospective cohort study and adheres to the "strengthening the reporting of observational studies in epidemiology (STROBE) statement" [15] and was conducted at a European academic level 1 trauma centre.

\section{Participants}

Patients who suffered a PHF that required surgical treatment were eligible to be included in this study. Only patients who suffered type B fractures following the AO/OTA fracture classification [16] who were treated with PHILOS were included in this study. Patients were stratified either to Group MIPO or Group ORIF. The treatment strategy based on the preference of the leading surgeon (FA). The senior surgeon (FA) was the leading surgeon in all cases.

All patients were strictly treated following the PHILOS guidelines provided by DePuy Synthes (Oberdorf, Switzerland). Group ORIF was treated following a delto-pectoral approach with open reduction and PHILOS.

The approach in Group MIPO included an anterolateral deltoid split proximally and a lateral approach to the humerus distally. The correct approach at the distal site was verified under the fluoroscope. Patients were followed-up 6 weeks, 3 months, 6 months and 12 months after the surgery as part of the routine clinical aftercare. Patients who did not complete the follow-up at our clinic, had secondary intervention at the humerus or suffered from multiple injuries of the arm were excluded from this analysis.

\section{Variables and definitions}

Outcome variables include complications and range of motion (ROM). Complications include postoperative nerve injury that was stratified to sensory or senso-motoric damage, injuries to the rotator cuff, and soft tissue complications including superficial or deep infection. Complications that were documented within 30 days after surgery were included in this study. The range of motion was quantified 12 months following the surgical intervention. To approximate clinical functionality ROM was quantified during the routine clinical follow-up and verified by the leading surgeon (FA). Functionality includes flexion above $90^{\circ}$ and the Apley scratch test, where patients were asked to touch their lumbar spine area during adduction, internal rotation, and retroversion in the shoulder joint. The radiation time during surgery was collected from the fluoroscopic device. All surgeries were performed with the same setting and the same device.

Radiologic union was assessed 12 months after surgery. A nonunion was defined when at least the fracture line was still visible without any signs of callus formation.

Patients' comorbidities were summarised according to Charlson comorbidity index (CCI) [17]. Trauma energy was stratified to low, medium, and high according to the definition by Tscherne [18]. Displacement was defined following the Neer classification [19].

\section{Data sources and study size}

Data were extracted from the electronic medical records and based on clinical measurements that were taken during routine clinical work. This study includes a maximal available dataset; therefore, a formal sample size calculation was not performed.

\section{Statistical analysis}

Continuous variables are summarised as the mean and standard deviation (SD), and categorical as number (n) and percentage. Group comparison was performed depending on distribution with the Students $t$ test, Mann-Whitney $U$ test, or depending on variable structure with the chi-square test. The level of significance was set at a $p$ value $<0.05$. All statistical analyses were performed using the $\mathrm{R}$ system for statistical computing and graphics, version 4.0.4 (R Core Team, 2021).

\section{Results}

\section{Participants and descriptive data}

During the 10 year period, 1488 patients were treated due to a fracture of the shoulder joint. Of these, 418 (28.1\%) required surgical treatment of a PHF. In total, 198 (47.4\%) fractures were classified as AO type B, 149 (75.3\%) were stratified in Group ORIF, and 49 (24.7\%) in Group MIPO (Fig. 1).

The mean age of the study population was 64.6 (SD 17.8) years and included $123(62.1 \%)$ female patients. The mean CCI was 2.8 (SD 2.3) points ranging from 0 to 10 points. Most patients $(n=153,77.3 \%)$ suffered a ground-level fall 


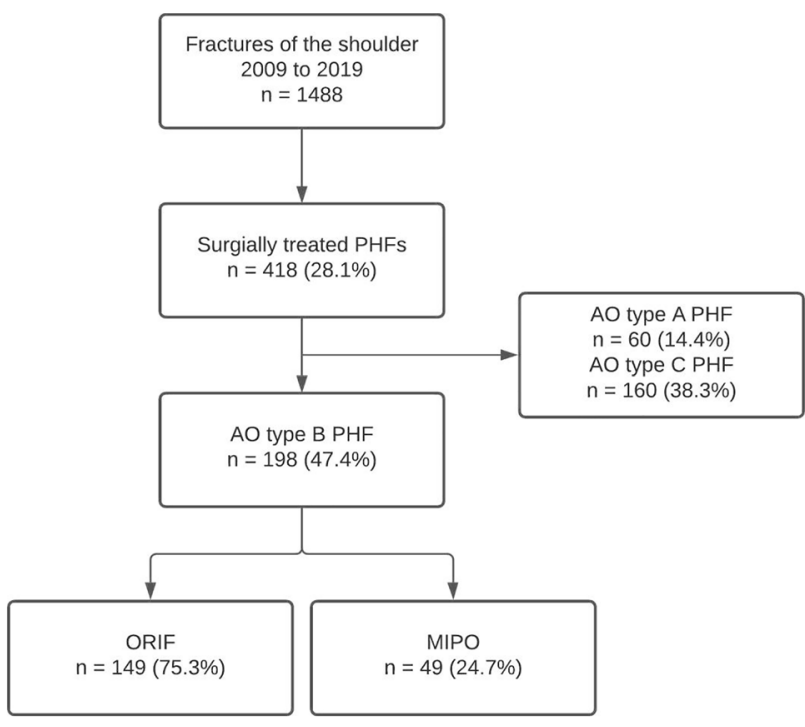

Fig. 1 Flow diagram of included patients. $P H F$ proximal humerus fracture, $A O$ Arbeitsgemeinschaft für Osteosynthesefragen, ORIF open reduction internal fixation, $M I P O$ minimal invasive plate osteosynthesis

representing low-energy trauma mechanism and $21(10.6 \%)$ patients suffered from an additional dislocation in the shoulder joint. The duration of surgery and radiation time where comparable in both groups (Table 1).

\section{Complications following surgical treatment of PHFs}

One patient $(2.0 \%)$ in Group MIPO reported persistent neuronal pain at rest as well as in motion for longer than 3 months, while another patient reported wound dehiscence. In Group ORIF, 15 (10.1\%) patients reported a complication. Five $(3.4 \%)$ patients reported transient postoperative nerve injury, three $(8.7 \%)$ reported injuries of the axillary nerve, and two (1.3\%) injuries of the radial nerve. In total, six $(4.0 \%)$ reported soft tissue complication. The remaining four $(2.6 \%)$ required reoperation based on screw perforation, and secondary humeral head necrosis. While not statistically significant, the rate of complications in Group ORIF was 2.6 (95\% CI 0.6-11.7) times higher when compared with Group MIPO. In total, 28 patients $(14.1 \%)$ had signs of radiological nonunion 12 months after surgery (2, 2.6\% Group MIPO vs. $26,10.6 \%$ Group ORIF, $p=0.049$ ).

\section{Range of motion}

The degree of internal and external rotation, adduction and extension were comparable amongst the groups at 12 months after surgery. Abduction was higher in Group MIPO (88.9 ${ }^{\circ}$, SD $4.2^{\circ}$ vs. $82.2^{\circ}$, SD $\left.16.2^{\circ}, p=0.035\right)$ as was elevation $\left(140.8^{\circ}, \mathrm{SD} 40.3^{\circ}\right.$ vs. $\left.93.1^{\circ}, \mathrm{SD} 68.9^{\circ}, p=0.001\right)$. Patients in Group MIPO had a further higher documented range of flexion $\left(139.1^{\circ}, \mathrm{SD} 30.2^{\circ}\right.$ vs. $\left.115.0^{\circ}, \mathrm{SD} 42.2^{\circ}, p=0.005\right)$.
Table 1 Demographics of study population (Type B fractures)

\begin{tabular}{llll}
\hline & ORIF & MIPO & $p$ value \\
\hline$n$ & 149 & 49 & \\
Age [years], mean (SD) & $64.84(17.95)$ & $63.86(17.84)$ & 0.742 \\
Female gender, $n(\%)$ & $92(62.2)$ & $31(63.3)$ & 1 \\
BMI [kg/m ${ }^{2}$ ], mean (SD) & $26.86(7.76)$ & $24.62(6.10)$ & 0.257 \\
CCI [points], mean (SD) & $2.71(2.10)$ & $3.18(2.34)$ & 0.187 \\
Trauma energy, $n(\%)$ & & & 0.266 \\
Low & $111(74.5)$ & $42(85.7)$ & \\
Medium & $21(14.1)$ & $4(8.2)$ & 0.316 \\
High & $17(11.4)$ & $3(6.1)$ & \\
Dislocation, $n(\%)$ & & $46(93.9)$ & \\
None & $131(87.9)$ & $0(0.0)$ & 0.432 \\
Subluxation & $6(4.0)$ & $3(6.1)$ & \\
Luxation & $12(8.1)$ & $49(100)$ & \\
Prim. Nerve injury, $n(\%)$ & & $0(0.0)$ & 0.141 \\
None & $144(96.6)$ & $0(0.0)$ & 0.702 \\
Sensory & $3(2.0)$ & $108.02(43.36)$ & $181.4(131.6)$ \\
Motoric & $2(1.3)$ & & \\
Duration of surgery [min], mean (SD) & $121.33(52.56)$ & $191.1(165.4)$ & \\
Duration of radiation during surgery [s], mean & & \\
(SD) & & & \\
\hline
\end{tabular}

$n$ number, $B M I$ body mass index, $C C I$ Charlson comorbidity index, ORIF open reduction internal fixation, $M I P O$ minimal invasive plate osteosynthesis 
Patients in Group MIPO had an 8.2 (95\% CI 2.4-27.7) times higher change to be able to flex the shoulder joint above $90^{\circ}$ at 12 months after surgery, when compared with Group ORIF. Similarly, Group MIPO had a 10.2 (95\% CI 2.4-43.7) times higher chance of reaching the lumbar vertebral body 1 (L1) or higher during the Apley scratch test when compared with Group ORIF (Table 2).

\section{Discussion}

MIPO represents a safe and effective alternative to ORIF as a surgical treatment strategy for PHFs [20, 21]. The aim of this study was to compare complication rates and the range of motion in patients who had a surgical treatment of type B PHFs using either the MIPO or ORIF techniques. This study revealed the following points:

- The postoperative complication rate was comparable between the groups.

- The functional outcome 12 months after surgery was better in Group MIPO when compared with Group ORIF.

- ORIF had higher risk for nonunion 12 months after surgery.

The increase rate of nonunion in Group ORIF might base on the periosteal stripping and the soft tissue handling around the fracture $[22,23]$. The rate of nonunion in the present study population is comparable with the literature $[22,23]$. This supports the fact, that adequate soft tissue handling and cautious treatment of the periosteum might support bone healing.

No postoperative radial nerve injury was observed in Group MIPO, in contrast to two cases (1.3\%) in Group ORIF. Iatrogenic radial nerve injury has been reported to range from 5.1 to $17.6 \%$ in ORIF of humerus shaft fractures [24, 25], which can also occur in MIPO techniques and during the treatment of PHF. Techniques for axillary nerve protections include visualization and tactile protection and have reduced the odds for iatrogenic axillary nerve damages

Table 2 Odds ratio of outcome measures MIPO (ref.) vs. ORIF

\begin{tabular}{llll}
\hline & OR & $95 \%$ CI & $p$ value \\
\hline Complications & 2.6 & $0.6-11.7$ & 0.252 \\
Postop. Nerve lesion & 0.9 & $0.1-9.7$ & 1 \\
Soft tissue complications & 2 & $0.2-17.2$ & 1 \\
Radiologic nonunion 12 months & 4.5 & $1.1-19.5$ & 0.035 \\
$\quad$ after surgery & & & \\
Flexion above 90 & 8.2 & $2.4-27.7$ & $<0.001$ \\
Apley scratch test L1 and higher & 10.2 & $2.4-43.7$ & $<0.001$ \\
\hline
\end{tabular}

$O R$ odds ratio, $C I$ confidence interval substantially [26]. The rate of wound infection after ORIF is reported to range from 1.9 [27] to $3.8 \%$ [28] and 4\% [4], which is comparable to the presented results. The rate of wound infection following MIPO was lower in the presented study (2.0\%). It is well known that minimally invasive surgery is associated with a lower rate of soft tissue infection [29]. The rate of avascular necrosis is reported to range from 5.5 to $10 \%[30,31]$, which is comparable to the presented results. Comparable to other reports [14], Group MIPO did not present with avascular necrosis of the humeral head, a complication that still can occur following MIPO [32].

Several studies investigated pain following MIPO or ORIF in PHFs. Some studies reported comparable VAS [33], while others documented less pain following MIPO [34]. The improved pain scores facilitates physiotherapeutic advancements and might, therefore, increase range of motion. Furthermore, the soft tissue preserving technique of MIPO favours bone healing and promotes improved functionality [35]. It has been reported that the rate of bone healing after MIPO is higher when compared with ORIF [23]. The minimal soft tissue disruption allows the earlier mobilisation of the shoulder, which further promotes healing and range of motion [36]. This might be based on the preservation of periosteal bridges, which could represent the last link of perfusion to the articular fragment [36]. This might further support the observation of lower rates of avascular humerus head necrosis following MIPO. The advantages of functional outcome, blood loss, and postoperative pain following MIPO have indicated beneficial outcomes, while the rate of complications remains comparable in selected cases [37].

\section{Limitations}

This study presents with certain limitations. The sample size in the present study is comparable with studies investigating the effect of MIPO on proximal humerus fractures [38-40]. Compared to these studies, the present study population is selected very carefully and includes only type B PHFs in order to improve outcome comparability and reduce confounding. Second, one might argue that the measurements of range of motion are not-standardised and lack relevance. This study included range of motion as an outcome measure representing the most relevant functional motions. While functional scoring system might represent a wide variety of daily activities, they might mask certain limitations of movement. The outcome (radiation time, duration of surgery, complications, etc.) might be associated with the surgeon's experience [41]. To minimize this bias, this study only includes cases, where the senior surgeon (FA) acted as the leading and supervising surgeon. The leading surgeon performed critical steps of the procedures (e.g. reduction), 
while other steps might have been performed by residents or fellows during their training.

\section{Conclusion}

MIPO is a valid and safe alternative to ORIF in type B fractures. The complication rates are comparable; however, functional outcome and range of motion might be improved following MIPO. Further large-scale studies are warranted to investigate the benefits and limitations of these two treatment strategies.

Authors contribution LB and SH: collection of data, analysis and interpretation, data curation, writing original draft, rewriting, this work serves as the doctoral thesis for LB under the supervision of $\mathrm{SH}$; MZ and DdH: developing research idea, investigation, study design and study protocol; RP, VN and FA: senior consultant, critical review and revision of manuscript; HCP: sponsor investigator, supervision, critical revision and interpretation. All authors read and approved the final version of the manuscript.

Funding Open Access funding provided by Universität Zürich. No external funding sources where utilized for this study.

Data availability All data, materials, and codes are available upon reasonable request.

\section{Declarations}

Conflict of interest None of the authors report any conflicts of interest.

Ethical approval This study and the study protocol were approved by the institutional review board (BASEC 2018-00146).

Open Access This article is licensed under a Creative Commons Attribution 4.0 International License, which permits use, sharing, adaptation, distribution and reproduction in any medium or format, as long as you give appropriate credit to the original author(s) and the source, provide a link to the Creative Commons licence, and indicate if changes were made. The images or other third party material in this article are included in the article's Creative Commons licence, unless indicated otherwise in a credit line to the material. If material is not included in the article's Creative Commons licence and your intended use is not permitted by statutory regulation or exceeds the permitted use, you will need to obtain permission directly from the copyright holder. To view a copy of this licence, visit http://creativecommons.org/licenses/by/4.0/.

\section{References}

1. Court-Brown CM, Caesar B. Epidemiology of adult fractures: a review. Injury. 2006;37:691-7.

2. Schumaier A, Grawe B. Proximal humerus fractures: evaluation and management in the elderly patient. Geriatr Orthop Surg Rehab. 2018;9:2151458517750516.

3. Halvachizadeh S, Gröbli L, Berk T, et al. The effect of geriatric co-management (GC) in geriatric trauma patients treated in a level 1 trauma setting: a comparison of data before and after the implementation of a certified geriatric trauma centre. PLoS One. 2021;16:e0244554.

4. Pfeifer R, Kalbas Y, Coimbra R, et al. Indications and interventions of damage control orthopaedic surgeries: an expert opinion survey. J Shoulder Elb Surg. 2020;32:528-35.

5. Gaebler C, McQueen M, Court-Brown C. Minimally displaced proximal humeral fractures epidemiology and outcome in 507 cases. Acta Orthop Scand. 2003;74:580-5.

6. Berkes MB, Little MTM, Lorich DG. Open reduction internal fixation of proximal humerus fractures. Curr Rev Musculoskelet Med. 2013;6:47-56.

7. Maier D, Jaeger M, Izadpanah $\mathrm{K}$, et al. Proximal humeral fracture treatment in adults. J Bone Jt Surg. 2014;96:251-61.

8. Halvachizadeh S, Berk T, Rauer T, et al. Treatment of proximal humerus fractures in geriatric patients-can pathological DEXA results help to guide the indication for allograft augmentation? PLoS One. 2020;15:e0230789.

9. Berk T, Halvachizadeh S, Bellmann F, et al. Does the magnitude of injuries affect the outcome of proximal humerus fractures treated by locked plating (PHILOS)? Eur J Trauma Emerg Surg. 2020. https://doi.org/10.1007/s00068-020-01451-9.

10. Brunner F, Sommer C, Bahrs C, et al. Open reduction and internal fixation of proximal humerus fractures using a proximal humeral locked plate: a prospective multicentre analysis. J Orthop Trauma. 2009;23:163-72.

11. Frima $\mathrm{H}$, Michelitsch C, Beks RB, et al. Long-term follow-up after MIPO Philos plating for proximal humerus fractures. Arch Orthop Trauma Surg. 2019;139:203-9.

12. Acklin YP, Stoffel K, Sommer C. A prospective analysis of the functional and radiological outcomes of minimally invasive plating in proximal humerus fractures. Injury. 2013;44:456-60.

13. Falez F, Papalia M, Greco A, et al. Minimally invasive plate osteosynthesis in proximal humeral fractures: one-year results of a prospective multicentre study. Int Orthop. 2016;40:579-85.

14. Acklin YP, Jenni R, Walliser M, Sommer C. Minimal invasive PHILOS ${ }^{\circledR}$-plate osteosynthesis in proximal humeral fractures. Eur J Trauma Emerg Surg. 2009;35:35-9.

15. Von Elm E, Altman DG, Egger M, et al. The strengthening the reporting of observational studies in epidemiology (STROBE) statement: guidelines for reporting observational studies. Ann Intern Med. 2007;147:573-7.

16. Meinberg E, Agel J, Roberts C, et al. Fracture and dislocation classification compendium-2018. J Orthop Trauma. 2018;32:S1-10. https://doi.org/10.1097/BOT.0000000000001063.

17. Charlson ME, Pompei P, Ales KL, MacKenzie CR. A new method of classifying prognostic comorbidity in longitudinal studies: development and validation. J Chronic Dis. 1987;40:373-83.

18. Tscherne H, Regel G. Tscherne unfallchirurgie: trauma-management. Berlin: Springer-Verlag; 2013.

19. Neer CS 2nd. Displaced proximal humeral fractures. Orthop Trauma Dir. 2007;5:25-9.

20. Shin S-J, Sohn H-S, Do N-H. Minimally invasive plate osteosynthesis of humeral shaft fractures: a technique to aid fracture reduction and minimise complications. J Orthop Trauma. 2012;26:585-9.

21. Kobayashi M, Watanabe Y, Matsushita T. Early full range of shoulder and elbow motion is possible after minimally invasive plate osteosynthesis for humeral shaft fractures. J Orthop Trauma. 2010;24:212-6.

22. Changulani M. Comparison of the use of the humerus intramedullary nail and dynamic compression plate for the management of diaphyseal fractures of the humerus: reply to Subasi and Cebesoy. Int Orthop. 2008;32:141.

23. Kim JW, Oh C-W, Byun Y-S, et al. A prospective randomized study of operative treatment for non-comminuted humeral shaft 
fractures: conventional open plating versus minimal invasive plate osteosynthesis. J Orthop Trauma. 2015;29:189-94.

24. Paris H, Tropiano P, Chaudet H, et al. Fractures of the shaft of the humerus: systematic plate fixation. Anatomic and functional results in 156 cases and a review of the literature. Rev Chir Orthop Reparatrice Appar Mot. 2000;86:346-59.

25. Lim KE, Yap CK, Ong SC, et al. Plate osteosynthesis of the humerus shaft fracture and its association with radial nerve injury-a retrospective study in Melaka General Hospital. Med J Malaysia. 2001;56:8-12.

26. Laflamme GY, et al. Percutaneous humeral plating of fractures of the proximal humerus: results of a prospective multicenter clinical trial. J Orthopaedic Trauma. 2008;3:153-8.

27. Athwal GS, Sperling JW, Rispoli DM, Cofield RH. Acute deep infection after surgical fixation of proximal humeral fractures. J Shoulder Elb Surg. 2007;16:408-12.

28. Vijayvargiya M, Pathak A, Gaur S. Outcome analysis of locking plate fixation in proximal humerus fracture. J Clin Diagnostic Res. 2016;10:RC01.

29. Rancan M, Dietrich M, Lamdark T, et al. Minimal invasive long PHILOS $^{\circledR}$-plate osteosynthesis in metadiaphyseal fractures of the proximal humerus. Injury. 2010;41:1277-83.

30. Röderer G, Erhardt J, Graf M, et al. Clinical results for minimally invasive locked plating of proximal humerus fractures. J Orthop Trauma. 2010;24:400-6.

31. Beeres FJP, Hallensleben NDL, Rhemrev SJ, et al. Plate fixation of the proximal humerus: an international multicentre comparative study of postoperative complications. Arch Orthop Trauma Surg. 2017;137:1685-92.

32. Xu J, Zhang C, Wang T. Avascular necrosis in proximal humeral fractures in patients treated with operative fixation: a meta-analysis. J Orthop Surg Res. 2014;9:1-6.

33. Zhao L, Yang P, Zhu L, Chen A. Minimally invasive percutaneous plate osteosynthesis (MIPPO) through deltoid-pectoralis approach for the treatment of elderly proximal humeral fractures. BMC Musculoskelet Disord. 2017;18:1-6.

34. Bockmann B, Buecking B, Franz D, et al. Mid-term results of a less-invasive locking plate fixation method for proximal humeral fractures: a prospective observational study. BMC Musculoskelet Disord. 2015;16:1-7.

35. Garcia-Virto V, Santiago-Maniega S, Llorente-Peris A, et al. MIPO helical pre-contoured plates in diaphyseal humeral fractures with proximal extension. Surgical technique and results. Injury. 2021;52(2):117-304.

36. Bogner R, Hübner C, Matis N, et al. Minimally-invasive treatment of three-and four-part fractures of the proximal humerus in elderly patients. J Bone Jt Surg Br. 2008;90:1602-7.

37. Li F, Liu X, Wang F, et al. Comparison between minimally invasive plate osteosynthesis and open reduction-internal fixation for proximal humeral fractures: a meta-analysis based on 1050 individuals. BMC Musculoskelet Disord. 2019;20:1-11.

38. Kim Y-G, Park K-H, Kim J-W, et al. Is minimally invasive plate osteosynthesis superior to open plating for fixation of two-part fracture of the proximal humerus? J Orthop Surg. 2019;27:2309499019836156.

39. Fischer C, Frank M, Kunz P, et al. Dynamic contrast-enhanced ultrasound (CEUS) after open and minimally invasive locked plating of proximal humerus fractures. Injury. 2016;47:1725-31.

40. Zhang Z, Zhang G, Peng Y, et al. Modified minimally invasive approach and intra-osseous portal for three-part proximal humeral fractures: a comparative study. J Orthop Surg Res. 2018;13:1-8.

41. Helfen T, Siebenbürger G, Fleischhacker E, Biermann N, Böcker $\mathrm{W}$, Ockert B. Open reduction and internal fixation of displaced proximal humeral fractures. Does the surgeon's experience have an impact on outcomes? PloS One. 2018;13(11):e0207044. 